\title{
Determination of the thicknesses of pipe elbows on the basis of expressions resulting from EU-Directive
}

Received: 11 April 2014 / Accepted: 10 December 2014 / Published online: 27 December 2014

(C) The Author(s) 2014. This article is published with open access at Springerlink.com

\begin{abstract}
The relations to calculate the maximum value of relative strains, which occur in a process of bending of tubes on benders, in stretched layers of tubes, are presented in this work on the basis of EU-Directive concerning production of pressure equipment. It has been shown that for large deformations that occur during bending of the pipes on knees, logarithmic strain measures (real) and relative strain measures give different values of the relative distribution of strain and different wall thicknesses in the bending zone. Reverse expressions were also derived to calculate the initial wall thickness of the tube to be bent, in order to obtain the desired wall thickness of knee after bending.
\end{abstract}

Keywords EU-Directive · Required thicknesses of pipe elbows · Pipe bending processes . Relative and logarithmic measures of deformation - Required initial thickness of a bent tube

\section{Introduction}

According to the EU-Directive on requirements in manufacturing of pressure equipment presented in [1,2] the measure of relative deformation is used for calculating the value of deformation [3-9] and this is a measure of the maximum value of the longitudinal component of the strain state for the case of first-order simplification $[10,11]$. In this paper, suitable equations will be derived for the three main components of relative deformations, which, according to formula from [1,2], reach their maximal values. Then, the expression for the minimum value of the wall thickness in knee bending layer will be derived. During tube bending on knees of pipelines or other piping systems, great strains are created (several tens of percent) [10-14] and then used logarithmic measures (logarithmic measures are frequently used for large and small deformations in engineering practice) of strain will not be equal to the measures of the relative strain. Different values will be obtained from the use of these measures. It will cause significant differences in the calculations, which will be demonstrated in the examples.

When for calculating of the state of strain you use the expression contained in the EU-Directive, for the purpose of calculating the minimum (acceptable) wall thickness, according to simplification of the first-order $[10,11]$, it will result that these thicknesses will be smaller than the calculated respectively in the measures of logarithmic deformation. Conversely, the required minimum initial thicknesses of the pipes to be bent will be correspondingly larger than those calculated in measures of logarithmic strain. In consequence, it means

Z. Śloderbach · J. Pajak ( $\varangle)$

Faculty of Applications of Chemistry and Mechanics, Opole University of Technology,

Luboszycka 7, 45-036 Opole, Poland

E-mail: j.pajak@po.opole.pl

Tel.: 77/449-8757, 77/449-8758

Z. Śloderbach

E-mail: z.sloderbach@ po.opole.pl 
that calculated on this basis, respectively, the minimum values of wall thickness and the required minimum thickness of bent pipes will be safer (lower estimates), respectively, than calculated, respectively, values in logarithmic (real) strain measures. This may result in that calculated according to logarithmic measures, the thicknesses of pipe walls may not meet the required (desired) value for the thickness of the walls calculated on the basis of the expression (stated in EU-Directive $[1,2]$ ) used to determine the deformation of the bent pipe.

The EU-Directive [1,2] also contains an empirical expression to calculate the required minimum wall thickness in stretched and compressed layers of bent knees. These expressions are identical to the expressions respecting practical applications given in papers $[15,16]$, except that instead of calculated thickness, the actual thickness is used. The expressions may be useful and can provide some criteria for the selection of an appropriate method (technology) of pipe bending for components of pressure equipment (for example with using drawing or pushing, with or without a mandrel [17-23]). This applies in particular to the conditions and requirements used to qualify the process of tube bending and to assessment of bent pipes and elbows designed for elements such as water-tubular boilers, see [1,2]. Further discussion and development of this problem will be presented in Sect. 6.

The aim of this work was to draw attention to the possible consequences that may arise from the use of expressions for calculating the deformation and wall thickness of the bent tube according to the EU-Directive. Given relationship for calculating the deformation is expressed in measures of relative deformations, depending on the $d_{\text {ext }}$ and refers to the stretched layers. According to the given classification, this expression is equal to the modified expression for the longitudinal component for the first-order simplification given in [10,11], when at the points of stretched layers, the state of maximum deformation is reached, so when $\left(\alpha=\beta=\beta_{1}=0^{\circ}\right.$ and $k \alpha_{b}=180^{\circ}$ ), see [10-13] and Figs. 1 and 2. In paper [12], the derivation of expressions for generalized scheme of deformations was presented, taking into account the shift of neutral axis $y_{0}$ and using the concept of kinematically admissible plastic strain fields of plastic strain. Since the bent tube is a spacial element, three components of strain state were derived, longitudinal, circumferential, and radial (in thickness). The use of kinematically admissible fields of plastic deformation is a simplified, commonly applied method in the technological theory of plasticity. Tube bending is treated as a process dependent on the angle of bending $\alpha_{b}$ as a parameter. The equations obtained in this way very well describe the experimental results presented in [17], see [12].

The use in this work of the first-order simplification derived in [10] instead of generalized model of strain $[12,13]$ is due to the fact that the expression in EU-directive corresponds to the expression for maximum elongation component of deformation for this simplification. The other two components of deformation (circumferential and radial), which are not included in EU-Directive, are the same as in the generalized model [11].

The examples of calculation results in our work show that the values of relative strain, thinning of the wall knees, and the required minimum wall thickness of bent tube, calculated according to the expression contained in the EU-Directive, are higher than those obtained with the use of logarithmic strain measures (real measures). This may cause some problems in assessment of pipe bending technology and estimation of manufacturing of a knee.

On the other hand, logarithmic measures of strain, due to their practical meaning, are most often used to the description of strain state of many plastic forming processes, in engineering practice and for tube bending $[3-7,13,14,21]$.

\section{Basic assumptions and relations}

In papers [1,2], an expression to calculate the deformation in the stretched layers in the process of tube bending in accordance with EU-Directive is presented.

$$
O=\frac{d_{\mathrm{ext}}}{2 R_{\mathrm{m}}}
$$

where $O$-longitudinal (axially) tensile strain in relative terms; $R_{\mathrm{m}}$ is a mean bending radius, $R_{\mathrm{m}} \in\langle R-$ $\left.y_{0 \max } ; R\right\rangle$ (Śloderbach [11]).

The parameters of bending process are presented in Fig. 1. As it results from Fig 1, Eq. (1) takes into consideration the shift of the neutral bending axis by the mean bending radius $R_{\mathrm{m}}$, since in general $R \neq R_{\mathrm{m}}$. If $y_{0}=0$, then $R=R_{\mathrm{m}}$. There is no unique definition in the Directive for the $R_{\mathrm{m}}$. This could be for 


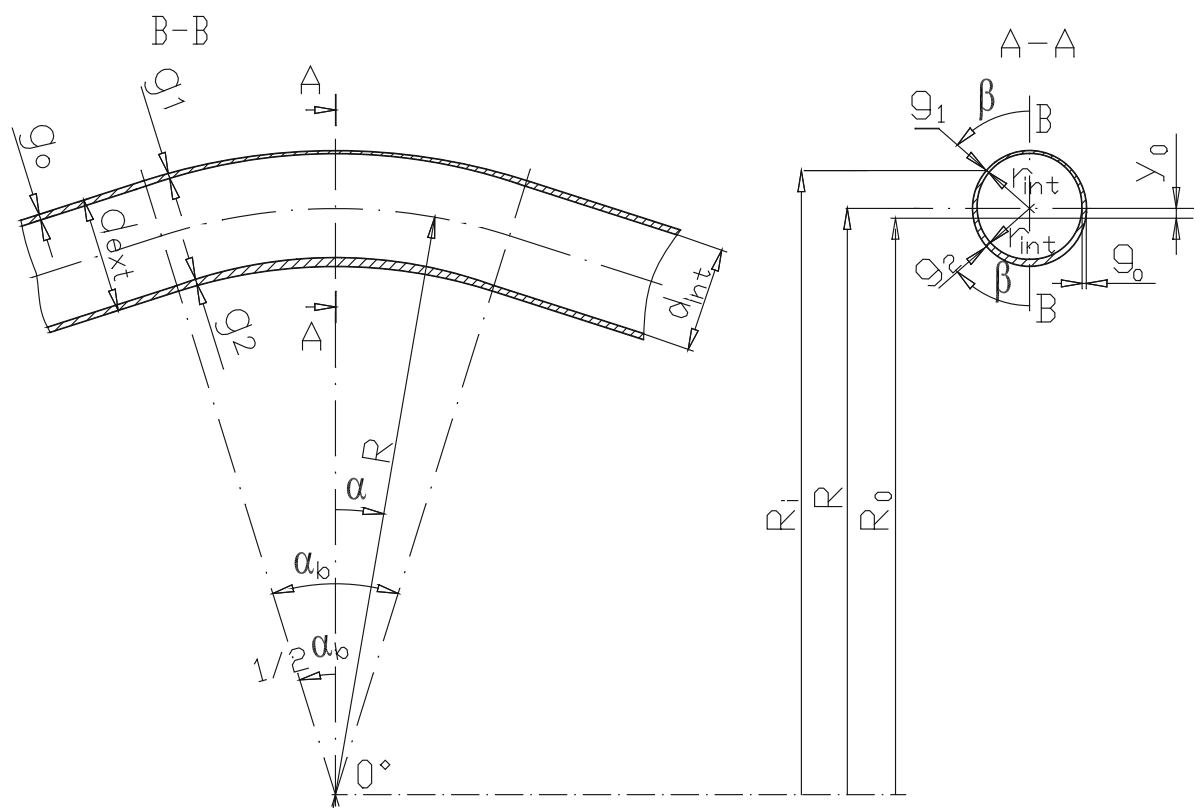

Fig. 1 Geometrical and dimensional quantities pertaining to tube-bending processes

example the arithmetic or geometric mean of bending radius $R$ and $\left(R-y_{0 \max }\right)$ or other value in the range $R_{\mathrm{m}} \in\left\langle R-y_{0 \max } ; R\right\rangle$.

Where on Fig. $1, d_{\mathrm{ext}}$ and $d_{\mathrm{int}}$ are external and internal diameter of a bent tube, $r_{\mathrm{int}}=d_{\mathrm{in}} / 2$

$g_{0}$-initial thickness of a bent tube,

$g_{i}$-actual thickness of a bend within the bending zone ( $i=1$ for elongated layers, $i=2$ for compressed layers),

$r_{\text {ext }}$ and $r_{\text {int }}$ - external and internal radius of a bent pipe,

$R$-bending radius,

$R_{0}$-radius of the neutral surface following bending, where $R_{0}=R-y_{0}$,

$R_{i}$ - larger actual radius of a bend associated with longitudinal strain,

$y_{0}$ - displacement of the neutral surface (axis) with respect to the initial position,

$\alpha$-actual angle of the bending zone determined at the principal bending plane and at planes parallel to it,

$$
\alpha \in\left\langle 0^{o} ; \frac{\alpha_{b}}{2}\right\rangle,
$$

where $\alpha_{b}$-active bending angle measured over the bending zone, $\alpha_{b} \in\left\langle 0^{\circ} ; 180^{\circ}\right\rangle$,

$\beta$ is actual angle determined at the planes perpendicular to the bending plane that $\beta \in\left\langle 0^{\circ}, 90^{\circ}\right\rangle$.

In this paper, the authors consider only cold bending of metal tubes of the assumed technological wall thickness $s^{*} \leq 0.10$ and maximal $d_{\mathrm{ext}}=160 \mathrm{~mm}$ (where $s^{*}=g_{0} / d_{\mathrm{ext}}, g_{0}$ and $d_{\mathrm{ext}}$-initial thickness and external diameter of the bent tube, respectively). In EU-Directive [1,2], the pressure tubes are assumed as thin-walled, when $s_{w}^{*} \leq 0.05$, where $s_{w}^{*}=g_{0} / d_{\text {int }}, d_{\text {int }}=d_{\text {ext }}-2 g_{0}$, then $s_{w}^{*}=s^{*} /\left(1-2 s^{*}\right)$.

\section{Expression for the displacement of the neutral axis}

In paper [24], author derived the following approximate expression for displacement of the neutral axis

$$
y_{0}=\frac{0.42}{\tilde{r}} r_{\mathrm{m}} .
$$

The extended expression, determining displacement of the neutral axis, valid for bending zones, was obtained by the first author and presented in [12], is:

$$
y_{0} \cong \lambda_{0} \frac{0.42}{\tilde{r}} r_{\mathrm{m}}\left(\cos (k \alpha)-\cos \left(k \frac{\alpha_{b}}{2}\right)\right),
$$




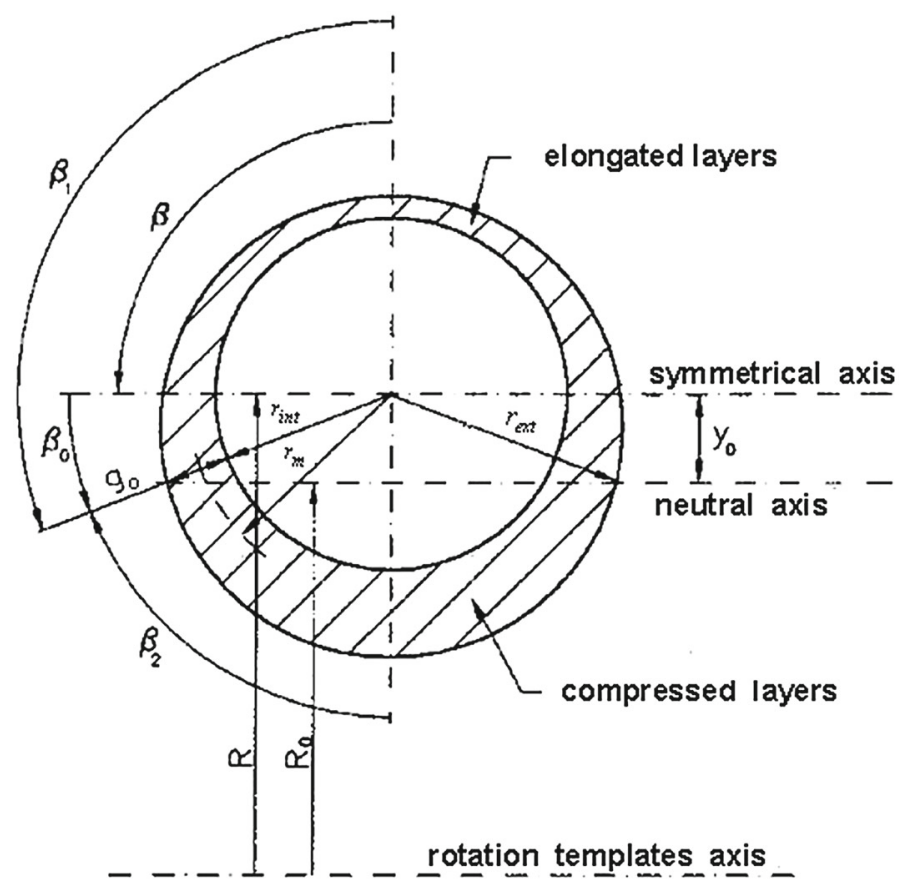

Fig. 2 Schematic picture of the elbow cross-section and its characteristic parameters

where $r_{\mathrm{m}}$-mean radius of the bent tube, $r_{m}=r_{\mathrm{int}}+g_{0} / 2$ (Fig. 2); $\tilde{r}$-relative radius of bending, $\tilde{r}=R / d_{\mathrm{ext}}$, $k$-technological-material coefficient dependent on the bent tube material and the applied bending technology, determining a bending zone range in the bent zone. This coefficient is defined during experiments, theoretically $k \in\langle 1 ; \infty)$. It seems that in the case of majority of metallic materials, it is sufficient when $k \in\langle 1 ; 6\rangle$. From the recognized tests and calculations, it even appears that $k \in\langle 1 ; 3\rangle$ (see e.g., [10-13,17,20]. In the case of more ductile, soft, plastic materials bent at elevated temperatures (hot, semi-hot or preheated bending) bent with a greater radius $R$ and at a more fitted expanding mandrel (segment, with an adjusted external diameter) with rich lubrication of the mandrel and the tube interior, then coefficient $k$ is lower (tends to the unit, $k \rightarrow 1$ ). Thus, it appears that coefficient $k$ allows to include (indirectly and in part) some effects of friction between the mandrel and the bent tube wall. For elbows bent to $180^{\circ}$, coefficient $k$ expresses a ratio of the bending angle $\alpha_{0}$ to a real value of the bending angle $\alpha_{b}$, i.e., $k=\alpha_{0} / \alpha_{b}$. When the bent angle $\left(\alpha_{0}=k \alpha_{b}=180^{\circ}\right)$, for example as in [9-12], then $k=180^{\circ} / \alpha_{b}$. If $\alpha_{0}=90^{\circ}$, then $2 \alpha_{0}=k \alpha_{b}=180^{\circ}$, when $\alpha=60$, then $3 \alpha_{0}=k \alpha_{b}=180^{\circ}$, etc. $\alpha_{0}$-bend angle (the angle by which a template or a farmer is rotated); in theory for spirals, $\alpha_{0} \in\left\langle 0^{\circ} ; \infty\right.$ ) but for the analyzed method $\alpha_{0} \in\left\langle 0^{\circ}, 180^{\circ}\right\rangle$. Obviously, within the bending zone, the two angles are equal $\left(\alpha_{0}=\alpha_{b}\right)$. When the plateau zone was formed, then $\left(\alpha_{0}=\alpha_{b}+\alpha_{\mathrm{pl}}\right)$, where $\alpha_{\mathrm{pl}}$-angle of a plateau zone [10-12].

Coefficient $\lambda_{0}$ determines characteristic technological-material parameters of the tube bending process, such as: a kind of the mandrel, a tube material, a shape of the template, and the flatter, strip pressure, clearances, forces of friction between the bent tube and the bending machine device, rigidity of the bending machine, a bending kind (cold, hot, self-hot, with preheating). From Eqs. (2) and (3), it appears that for very small bending radii $R \in\left\langle 0.5 \times d_{\text {ext }} ; 1 \times d_{\text {ext }}\right\rangle$ and more thin-walled tubes $\left(s_{w}^{*}<<0.05\right)$, the maximum displacement of the neutral axis can be equal $\sim 25 \%$ of a diameter value of the tube which is going to be bent. Greater displacements of the neutral axis can be caused by another bending technology because in the case of the considered ranges $\tilde{r}$ and $s_{w}^{*}$ tubes are often bent with use of a force which is opposite to the force rotating the template so as to obtain a suitable stress distribution in the cross section. From the extended Eq. (3) it also appears that displacement of the neutral axis is influenced not only by the bending radius and the tube thickness (thin-walled) but also by a suitable technology, bending parameters, and the tube material, as well. From Eq. (3), it also appears that there are three additional parameters determining displacement of the neutral axis and its position in the bending zone: the bending angle and the angle determining a position of the point in the bending zone, and the coefficient $k$. Thus, if $\left(\cos (k \alpha)=1\right.$ and $\left.\cos \left(k \frac{\alpha_{b}}{2}\right)=0\right)$, then $y_{0}=y_{\mathrm{o} \text { max }} \cong \lambda_{0} \frac{0,42}{\tilde{r}} r_{\mathrm{m}}$, see Eq. (3). 
In Fig. 2, $\beta_{1}$ and $\beta_{2}$-angle determined in elongated and compressed layers, $\sin \beta_{0}=y_{0} / r_{\mathrm{ext}} \approx y_{0} / r_{\mathrm{m}}$. Taking some additional calculations into account, in practice, it is recommended to limit the considered bending method to the radii $\left(R \geq 1.5 \times d_{\text {ext }}\right)$. Pressure tubes which are most often used in pipelines for power industry and other tube installations of the devices of power engineering are usually included into the range $\left(0.00<s_{w}^{*} \leq 0.125\right)$ or $\left(0.00<s^{*} \leq 0.10\right)$.

The introduced limitations concerning the tube bending parameters cause that, for example, the maximum (for instance for $R=1.0 \times d_{\text {ext }}, s_{w}^{*}=0.03$ and $\lambda_{0}=0.5$ ) relative (related to the external diameter of the bent tube) displacement of the neutral axis is $y_{0} / d_{\text {ext }} \approx 10 \%$. However, for some ranges $\left(R\right.$ and $\left.s_{w}^{*}\right)$ and bending technologies and tube materials, relationships which do not include displacement of the neutral axis $y_{0}$ can be applied for strain description. Thus, they were applied in [13] for precise description of fundamental experiments presented in [17]. The estimated maximum value $y_{0}$ can be in practice even lower owing to a suitable selection and setup of tooling of the bending machine, removal of clearances, a more plastic material for the bent tube, application of bending at elevated temperatures, increase in rigidity of the bending machine and so on. In the compressed layers, effects resulting from not unbounded upsetting may be less, they will be more intense along the perimeter of displacement of the bent tube material to the sides, upward and along the bent axis; it can cause lower values of the coefficient $\lambda_{0}$.

According to the assumptions that the derived expressions for strain components in tube bending processes are identified with plastic strains (it appears that in the angular measure elastic strains are related to the main bending angle equal to some degrees [10-13]) we obtain that $\varepsilon_{1}^{\prime}, \varepsilon_{2}^{\prime}, \varepsilon_{3}^{\prime}$ are relative components of plastic deformations for the first-order simplification and $\varphi_{1}^{\prime}, \varphi_{2}^{\prime}, \varphi_{3}^{\prime}$ are logarithmic components of plastic deformations for the first-order simplification. Since the bent pipe is spatial, proper analysis of the plastic strain requires the determination of three major components of strain. These components in relative and logarithmic measures according to symbolism accepted in mechanics of solids and according to designations used in papers of Śloderbach [10-13] when $\left(y_{0} \neq 0\right.$ and $\left.R=R_{\mathrm{m}}\right)$, after formally transformations have, for the case of first-order simplification [10,11], the following forms:

$$
\left\{\begin{array}{l}
\varepsilon_{1}^{\prime}=\frac{d_{\mathrm{ext}} \cos \beta_{1}\left(\cos (k \alpha)-\cos \left(k \frac{\alpha_{b}}{2}\right)\right)}{2 R_{\mathrm{m}}} \\
\varepsilon_{2}^{\prime}=\frac{d_{1 r}^{\prime}-d_{\mathrm{ext}}}{d_{\mathrm{ext}}}, \quad \varepsilon_{3}^{\prime}=\frac{g_{1 r}^{\prime}-g_{0}}{g_{0}}
\end{array}\right.
$$

and

$$
\left\{\begin{array}{l}
\varphi_{1}^{\prime}=\ln \frac{2 R_{\mathrm{m}}+d_{\mathrm{ext}} \cos \beta_{1}\left(\cos (k \alpha)-\cos \left(k \frac{\alpha_{b}}{2}\right)\right)}{2 R_{\mathrm{m}}}, \\
\varphi_{2}^{\prime}=\ln \frac{d_{1 l}^{\prime}}{d_{\mathrm{ext}}}, \quad \varphi_{3}^{\prime}=\ln \frac{g_{1 l}^{\prime}}{g_{0}},
\end{array}\right.
$$

where $d_{1 r}^{\prime}$ and $d_{1 l}^{\prime}$ - the outer minimum diameter of knee in stretched layers determined for the relative and logarithmic measures of strain, respectively, where: $d_{1 r}^{\prime}=d_{\text {int }}+2 g_{1 r}^{\prime}$ and $d_{1 l}^{\prime}=d_{\text {int }}+2 g_{1 l}^{\prime}, g_{1 r}^{\prime}$ and $g_{1 l}^{\prime}$ are minimum wall thickness of bent knee in tension layers determined for the relative and logarithmic measures of strain, respectively.

When $\left(\alpha=\beta_{1}=0^{\circ}\right.$ and $\left.k \alpha_{b}=180^{\circ}\right)$, then equations (4) $)_{1}$ and $(5)_{1}$ take their maximum values, as

$$
\varepsilon_{1}^{\prime}=\frac{d_{\mathrm{ext}}}{2 R_{\mathrm{m}}}, \varepsilon_{2}^{\prime}=\frac{d_{1 r}^{\prime}-d_{\mathrm{ext}}}{d_{\mathrm{ext}}}, \varepsilon_{3}^{\prime}=\frac{g_{1 r}^{\prime}-g_{0}}{g_{0}} .
$$

and

$$
\varphi_{1}^{\prime}=\ln \frac{2 R_{\mathrm{m}}+d_{\mathrm{ext}}}{2 R_{\mathrm{m}}}, \varphi_{2}^{\prime}=\ln \frac{d_{1 l}^{\prime}}{d_{\mathrm{ext}}}, \varphi_{3}^{\prime}=\ln \frac{g_{1 l}^{\prime}}{g_{0}},
$$

Expressions (4), (5), (6), and (7) are empirical relationships mutually arising from engineering definitions of measures of strains and adoption of incompressibility condition of plastically deformed materials. Incompressibility condition is valid for majority of metallic materials.

The equations for intensity of plastic strain for strain measures (4), (5), (6) and (7) are the followings:

$$
\left\{\begin{array}{l}
\varepsilon_{(i)}^{\prime}=\sqrt{\frac{2}{3}\left(\varepsilon_{1}^{, 2}+\varepsilon_{2}^{, 2}+\varepsilon_{3}^{, 2}\right)} \\
\varphi_{(i)}^{\prime}=\sqrt{\frac{2}{3}\left(\varphi_{1}^{, 2}+\varphi_{2}^{, 2}+\varphi_{3}^{, 2}\right)} .
\end{array}\right.
$$


The conditions of plastic incompressibility of the material have the following form:

$$
\left\{\begin{array}{l}
\varepsilon_{1}^{\prime}+\varepsilon_{2}^{\prime}+\varepsilon_{3}^{\prime}=0, \\
\varphi_{1}^{\prime}+\varphi_{2}^{\prime}+\varphi_{3}^{\prime}=0 .
\end{array}\right.
$$

Expression $(9)_{1}$, valid for small deformations $(<5 \%)$ is often used in practical technological applications of plasticity theory, see e.g., [3-9,24-26]. Here, we used it as the first step to show the problem of differences in calculating the strain in logarithmic and relative values. According to data $[11,13,17]$, for maximal deformations, the value of $\left(\varepsilon_{1}^{\prime}+\varepsilon_{2}^{\prime}+\varepsilon_{3}^{\prime}\right)$ is about $5 \%$ and was omitted in our empirical calculations.

Formulas for calculating the coefficients of the maximum thinning of tube wall with respect to its initial thickness are the following $[15,16,20]$ :

$$
\left\{\begin{array}{l}
K_{g^{\prime} r}=\frac{g_{0}-g_{1 r}^{\prime}}{g_{0}}, \\
K_{g^{\prime} l}=\frac{g_{0}-g_{11}^{\prime}}{g_{0}} .
\end{array}\right.
$$

\section{Calculation of the required minimum wall thickness}

Substituting components of relative plastic strain (6) and (7), respectively, to incompressibility conditions (9) 1 and $(9)_{2}$, after transformations, we obtain the following expression for the appropriate minimum wall thickness of the knee in the apex points of tension layers: $\left(\alpha=\beta_{1}=0^{\circ}\right.$ and $\left.k \alpha_{b}=180^{\circ}\right)$, for the case when $\left(y_{0} \neq 0\right.$ and $R \neq R_{\mathrm{m}}$ ). Hence

$$
\left\{\begin{array}{l}
g_{1 r}^{\prime}=g_{0}-\frac{g_{0} d_{\mathrm{ext}}^{2}}{2 R_{\mathrm{m}}\left(d_{\mathrm{ext}}+2 g_{0}\right)}, \\
g_{1 l}^{\prime}=-\frac{\left(d_{\mathrm{ext}}-2 g_{0}\right)}{4}+\sqrt{\left(\frac{d_{\mathrm{ext}}-2 g_{0}}{4}\right)^{2}+\frac{R_{\mathrm{m}} d_{\mathrm{ext}} g_{0}}{2 R_{\mathrm{m}}+d_{\mathrm{ext}}}},
\end{array}\right.
$$

where $g_{1 r}^{\prime}$ and $g_{1 l}^{\prime}$-minimum wall thickness of bent knee in tension layers determined for the relative and logarithmic measures of strain, respectively,

Example 1 Let the mean bending radius $R_{\mathrm{m}}=80 \mathrm{~mm}\left(R_{\mathrm{m}} \approx 1.8 \times d_{\mathrm{ext}}\right)$, and the dimensions of bent pipe: $\phi 44.5 \times 4.5 \mathrm{~mm}$. Based on equations (11) 1 and 2 we obtain:

$$
g_{1 r}^{\prime} \cong 3.459 \mathrm{~mm}, \quad g_{1 l}^{\prime} \cong 3.659 \mathrm{~mm} .
$$

These are calculated minimum wall thickness of the bent knee in stretch layers expressed in real (logarithmic) and relative measures of strain, respectively, obtained on the basis of the EU-Directive [1,2]. Corresponding to the above calculations, thinning coefficients have the following values $K_{g / r} \cong 0.231, K_{g / l} \cong 0.187$.

Based on the above results and on the data from Table 1, we obtain the following inequality

$$
g_{1 r}^{\prime}<g_{1 l}^{\prime},
$$

Thus, on the basis of formulas (10), it results that

$$
K_{g^{\prime} r}>K_{g^{\prime} l}
$$

For the case of large strains, see e.g., [3,10-13,26-31], and such strains occur during bending of tubes in stretched layers (which are valid for each bend radius $R$ or $R_{\mathrm{m}}$ and geometric dimensions of bent tube), and based on inequalities (12) and (13), the following inequalities occur in strains, which are equivalent to inequality (12)

$$
\varepsilon_{1}^{\prime}>\varphi_{1}^{\prime},\left|\varepsilon_{2}^{\prime}\right|>\left|\varphi_{2}^{\prime}\right|,\left|\varepsilon_{3}^{\prime}\right|>\left|\varphi_{3}^{\prime}\right|
$$

and

$$
\varepsilon_{(i)}^{\prime}>\varphi_{(i)}^{\prime} .
$$


Table 1 Computed minimum wall thickness values within stretched layers $\left(\lambda_{1}=1\right)$ for the simplified method of first-order, expressed through logarithmic and relative strains $\tilde{r}$ as functions of mean bending radius $R_{\mathrm{m}}$

\begin{tabular}{llll}
\hline$R_{\mathrm{m}}(\mathrm{mm})$ & $R_{\mathrm{m}}\left(\tilde{r} \times d_{\mathrm{ext}}\right)$ & $g_{1 l}^{\prime}(\mathrm{mm})$ & $g_{1 r}^{\prime}(\mathrm{mm})$ \\
\hline 22.250 & $R_{\mathrm{m}}=0.50 \times d_{\mathrm{ext}}$ & 2.475 & 0.757 \\
33.375 & $R_{\mathrm{m}}=0.75 \times d_{\mathrm{ext}}$ & 2.908 & 2.005 \\
44.500 & $R_{\mathrm{m}}=1.0 \times d_{\mathrm{ext}}$ & 3.188 & 2.629 \\
55.625 & $R_{\mathrm{m}}=1.25 \times d_{\mathrm{ext}}$ & 3.384 & 3.003 \\
66.750 & $R_{\mathrm{m}}=1.50 \times d_{\mathrm{ext}}$ & 3.529 & 3.252 \\
77.875 & $R_{\mathrm{m}}=1.75 \times d_{\mathrm{ext}}$ & 3.641 & 3.431 \\
80.000 & $R_{\mathrm{m}} \cong 1.80 \times d_{\mathrm{ext}}$ & 3.659 & 3.459 \\
89.000 & $R_{\mathrm{m}}=2.0 \times d_{\mathrm{ext}}$ & 3.729 & 3.564 \\
100.125 & $R_{\mathrm{m}}=2.25 \times d_{\mathrm{ext}}$ & 3.801 & 3.668 \\
111.250 & $R_{\mathrm{m}}=2.50 \times d_{\mathrm{ext}}$ & 3.861 & 3.751 \\
122.375 & $R_{\mathrm{m}}=2.75 \times d_{\mathrm{ext}}$ & 3.911 & 3.819 \\
133.500 & $R_{\mathrm{m}}=3.00 \times d_{\mathrm{ext}}$ & 3.954 & 3.876 \\
144.625 & $R_{\mathrm{m}}=3, .25 \times d_{\mathrm{ext}}$ & 3.991 & 3.924 \\
155.750 & $R_{\mathrm{m}}=3.50 \times d_{\mathrm{ext}}$ & 4.024 & 3.965 \\
178.000 & $R_{\mathrm{m}}=4.00 \times d_{\mathrm{ext}}$ & 4.077 & 4.032 \\
200.250 & $R_{\mathrm{m}}=4.50 \times d_{\mathrm{ext}}$ & 4.120 & 4.084 \\
222.500 & $R_{\mathrm{m}}=5.00 \times d_{\mathrm{ext}}$ & 4.155 & 4.126 \\
\hline
\end{tabular}

The other dimensions of the tube were assumed as follows: $d_{\mathrm{ext}}=44.5 \mathrm{~mm}$ and $g_{0}=4.5 \mathrm{~mm}$

The above examples of computational results show that the values of relative strains and the required minimum wall thickness calculated according to the EU-Directive are higher than those obtained with the use of logarithmic (real) strain measures. On the other hand, it is known that logarithmic measures of strain, due to their practical properties, are usually used to describe the state of strain in several plastic forming processes, including the pipe bending $[10-13,17-20,26]$. This fact may cause some problems in designing and technology, and also in operating and resistance.

The values of thinning coefficient of the wall thickness, calculated in Example 1, in all cases exceed the value of acceptable thinning, which, according to Korzemski [20] for this outer diameter of tube, bent with radius $R_{\mathrm{m}} \geq 3 \times d_{\text {ext }}$, is equal $K_{\text {grall }}=0.08$. It results from the fact that bending with radius $R_{\mathrm{m}} \cong 1.8 \times d_{\text {ext }}$ (as in Example 1) is "sharper". For knees made of thin-walled metal on appropriate benders (with rotating template and during upsetting [10-13,17-24] and bent with radius $R_{\mathrm{m}} \geq 1.2 \times d_{\mathrm{ext}}$, the thinning of wall can be smaller than $15 \%\left(K_{g \prime}<0.15\right)$. From formulas $(11)_{1,2}$, it also results that if bending radius $R_{\mathrm{m}}$ tends to infinity, then the values $g_{1 r}^{\prime}$ and $g_{1 l}^{\prime}$ tend to value $g_{0}$, respectively, and that means the lack of bending effect.

\section{Determination of the required initial thickness of bent tube}

This is the inversed problem to the one considered in the previous section. The required (desired) wall thickness $g_{1}$ that fulfills, for example, resistance and construction conditions, technological and operational requirements, requirements EU-PN (PN-Polish Standarts) or regulations of the UDT will be discussed (Office of the Polish Technical Supervision, see $[15,16]$ ). Searched is the initial (starting) required thickness $g_{0}$ of wall of tube to be bent.

Substituting the components of plastic strains, relative (6) and logarithmic (7), respectively, to incompressibility condition $(9)_{1}$ and $(9)_{2}$, after transformations, we obtain the following expression for appropriate initial (starting) required thickness of the wall of tube to be bent in the stretched layers:

$$
g_{0 r}^{\prime}=\frac{-\left[2 R_{\mathrm{m}}\left(d_{\mathrm{ext}}-2 g_{1}\right)-d_{\mathrm{ext}}^{2}\right]}{8 R_{\mathrm{m}}}+\frac{\sqrt{\left[2 R_{\mathrm{m}}\left(d_{\mathrm{ext}}-2 g_{1}\right)-d_{\mathrm{ext}}^{2}\right]^{2}+32 R_{\mathrm{m}}^{2} d_{\mathrm{ext}} g_{1}}}{8 R_{\mathrm{m}}},
$$

and

$$
g_{0 l}^{\prime}=\frac{g_{1}\left(d_{\mathrm{ext}}+2 g_{1}\right)\left(2 R_{\mathrm{m}}+d_{\mathrm{ext}}\right)}{2\left[R_{\mathrm{m}}\left(d_{\mathrm{ext}}+2 g_{1}\right)+d_{\mathrm{ext}} g_{1}\right]},
$$

where $g_{0 r}^{\prime}$ and $g_{0 l}^{\prime}$-required initial wall thickness values expressed through relative and logarithmic strains, $g_{1}-\mathrm{a}$ required (desired) minimum wall thickness of the knee in the apex point of stretched layers. 
Table 2 Calculated initial thickness of the tube walls for the simplified method of first-order, expressed through true and relative strains $\tilde{r}$ as functions of mean bending radius $R_{\mathrm{m}}$, when the required minimum thickness of the bend elbow wall is $g_{1}=4.5 \mathrm{~mm}$ and the outer diameter of the tube is $d_{\mathrm{ext}}=44.5 \mathrm{~mm}$

\begin{tabular}{|c|c|c|c|}
\hline$R_{\mathrm{m}}(\mathrm{mm})$ & $R_{\mathrm{m}}\left(\tilde{r} \times d_{\mathrm{ext}}\right)$ & $g_{0 l}^{\prime}(\mathrm{mm})$ & $g_{0 r}^{\prime}(\mathrm{mm})$ \\
\hline 22.250 & $R_{\mathrm{m}}=0.50 \times d_{\mathrm{ext}}$ & 7.704 & 12.506 \\
\hline 33.375 & $R_{\mathrm{m}}=0.75 \times d_{\mathrm{ext}}$ & 6.744 & 9.364 \\
\hline 44.500 & $R_{\mathrm{m}}=1.00 \times d_{\mathrm{ext}}$ & 6.226 & 7.228 \\
\hline 55.625 & $R_{\mathrm{m}}=1.25 \times d_{\mathrm{ext}}$ & 5.903 & 6.516 \\
\hline 66.750 & $R_{\mathrm{m}}=1.50 \times d_{\mathrm{ext}}$ & 5.680 & 6.095 \\
\hline 77.875 & $R_{\mathrm{m}}=1.75 \times d_{\mathrm{ext}}$ & 5.520 & 5.818 \\
\hline 80.000 & $R_{\mathrm{m}} \cong 1.80 \times d_{\mathrm{ext}}$ & 5.495 & 5.775 \\
\hline 89.000 & $R_{\mathrm{m}}=2.00 \times d_{\mathrm{ext}}$ & 5.398 & 5.622 \\
\hline 100.125 & $R_{\mathrm{m}}=2.25 \times d_{\mathrm{ext}}$ & 5.302 & 5.477 \\
\hline 111.250 & $R_{\mathrm{m}}=2.50 \times d_{\mathrm{ext}}$ & 5.224 & 5.364 \\
\hline 122.375 & $R_{\mathrm{m}}=2.75 \times d_{\mathrm{ext}}$ & 5.160 & 5.275 \\
\hline 133.500 & $R_{\mathrm{m}}=3.00 \times d_{\mathrm{ext}}$ & 5.107 & 5.203 \\
\hline 144.625 & $R_{\mathrm{m}}=3.25 \times d_{\mathrm{ext}}$ & 5.061 & 5.143 \\
\hline 155.750 & $R_{\mathrm{m}}=3.50 \times d_{\mathrm{ext}}$ & 5.022 & 5.092 \\
\hline 178.000 & $R_{\mathrm{m}}=4.00 \times d_{\mathrm{ext}}$ & 4.958 & 5.011 \\
\hline 200.250 & $R_{\mathrm{m}}=4.50 \times d_{\mathrm{ext}}$ & 4.908 & 4.950 \\
\hline 222.500 & $R_{\mathrm{m}}=5.00 \times d_{\mathrm{ext}}$ & 4.866 & 4.906 \\
\hline
\end{tabular}

Example 2 Let the mean bending radius $R_{\mathrm{m}}=80 \mathrm{~mm}\left(R_{\mathrm{m}} \approx 1.8 \times d_{\mathrm{ext}}\right)$, bending angle $\left(k \alpha_{b}=180^{\circ}\right)$, outer diameter of tube $d_{\mathrm{ext}}=44.5 \mathrm{~mm}$, and the required wall thickness of the knee in the apex (middle) point of stretched layers $g_{1}=4.5 \mathrm{~mm}$. Then, on the basis of formulas (16) and (17) after calculations, we obtain: $g_{0 r}^{\prime}=5.775 \mathrm{~mm}$ and $g_{0 l}^{\prime}=5.495 \mathrm{~mm}$.

Required initial wall thickness values obtained, respectively, for logarithmic and relative measures of strain are computed, depending on values of outer diameter of bent tube on the basis of EU-Directive.

On the basis of these results and the data from Table 2, we obtain the following inequality:

$$
g_{0 r}^{\prime}>g_{0 l}^{\prime}
$$

The method of using the relations derived in this section is as follows: for given parameters of bending described with average bending radius $R_{\mathrm{m}}$ and for given geometric dimensions of tube to be bent $\left(l \times d_{\mathrm{ext}}\right)$ and for required value of wall thickness of bent knee $g_{1}$, required value of initial thickness of tube to be bent is determined on the basis of expressions (16) and (17). Then, the tube with the first value of wall thickness bigger than this initial value should be chosen from the manufacturer's catalog and in accordance with EU Standards. From formulas (16) and (17), it also results that when bending radius $R_{\mathrm{m}}$ tends to infinity, then the values of $g_{0 \mathrm{r}}^{\prime}$ and $g_{0 l}^{\prime}$ tend to $g_{1}$ and that means the lack of results of bending.

\section{Results and discussion}

Changes in the minimum thickness of the bent knee $\left(g_{1 r}^{\prime}\right.$ and $\left.g_{1 l}^{\prime}\right)$ depending on the average bending radius $R_{\mathrm{m}}$, for bent tube with dimensions $\phi 44.5 \times 4.5(\mathrm{~mm})$, with the use of relative and logarithmic measures of strain, respectively, are presented in Table 1 and Fig. 3. As can be visible from the plots, when bending radius $R_{\mathrm{m}}$ decreased, then the differences in thicknesses are increased since the differences in strain values are greater. The use of relative and logarithmic measures of strain causes the formation of significant differences in the calculations. It is on the contrary, when the bending radius $R_{\mathrm{m}}$ increases. When bending radius $R_{\mathrm{m}}$ strives to infinity, then calculated thicknesses $\left(g_{1 \mathrm{r}}^{\prime}\right.$ and $\left.g_{11}^{\prime}\right)$ strive, respectively, to thickness $g_{0}\left(g_{0}=4.5 \mathrm{~mm}\right)$ and that means the lack of bending.

The use of relative measures of the components of strain (according to EU-Directive) results in that the calculated strains are greater than those calculated with the use of logarithmic strain measures. Therefore, the calculated minimum wall thickness of the bent knee, calculated with this method, will be smaller. That means that from operational and resistance points of view, such estimations will be safer. Thus, it might be concluded that during calculation of the wall thickness on the basis of expression (1) from the EU-Directive, 


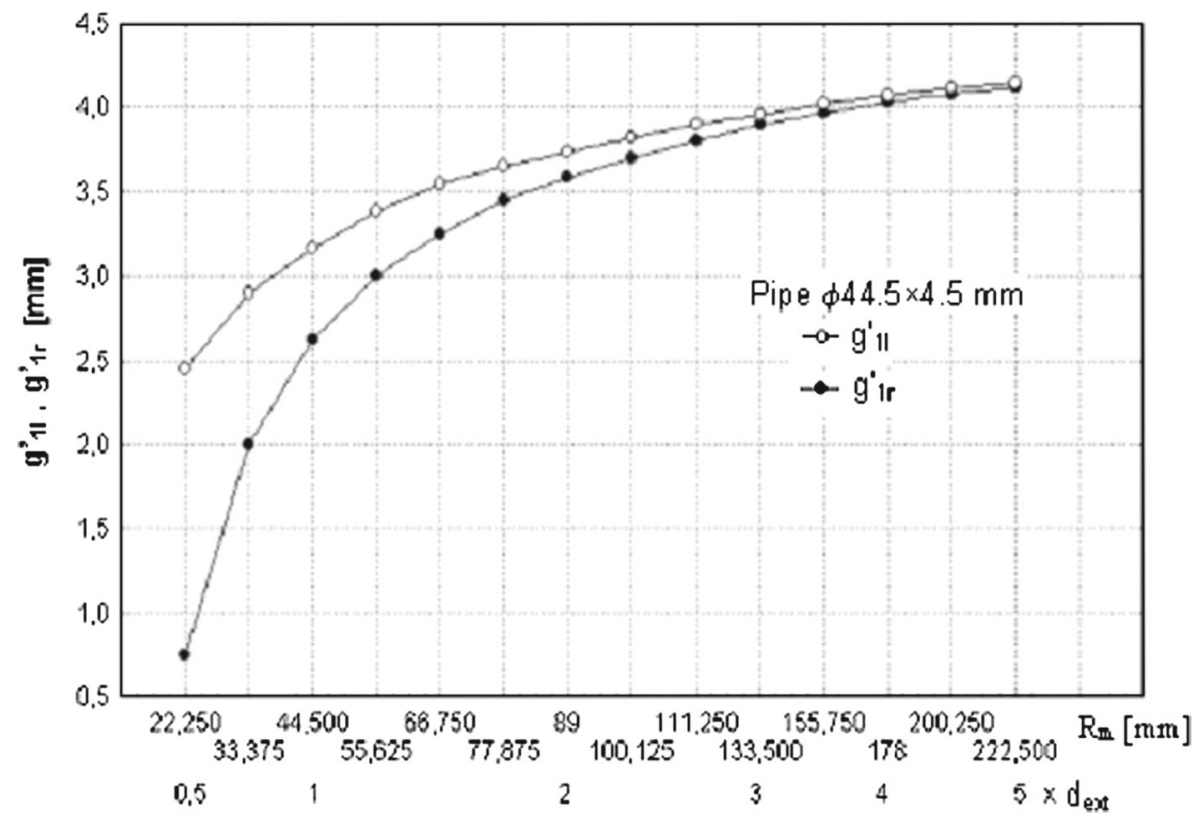

Fig. 3 Variation of the minimum wall thickness values $\left(g_{l r}^{\prime}, g_{l w}^{\prime}\right)$ of a bend with bending radius $R_{\mathrm{m}}$ for $\phi 44.5 \times 4.5 \mathrm{~mm}$ pipe

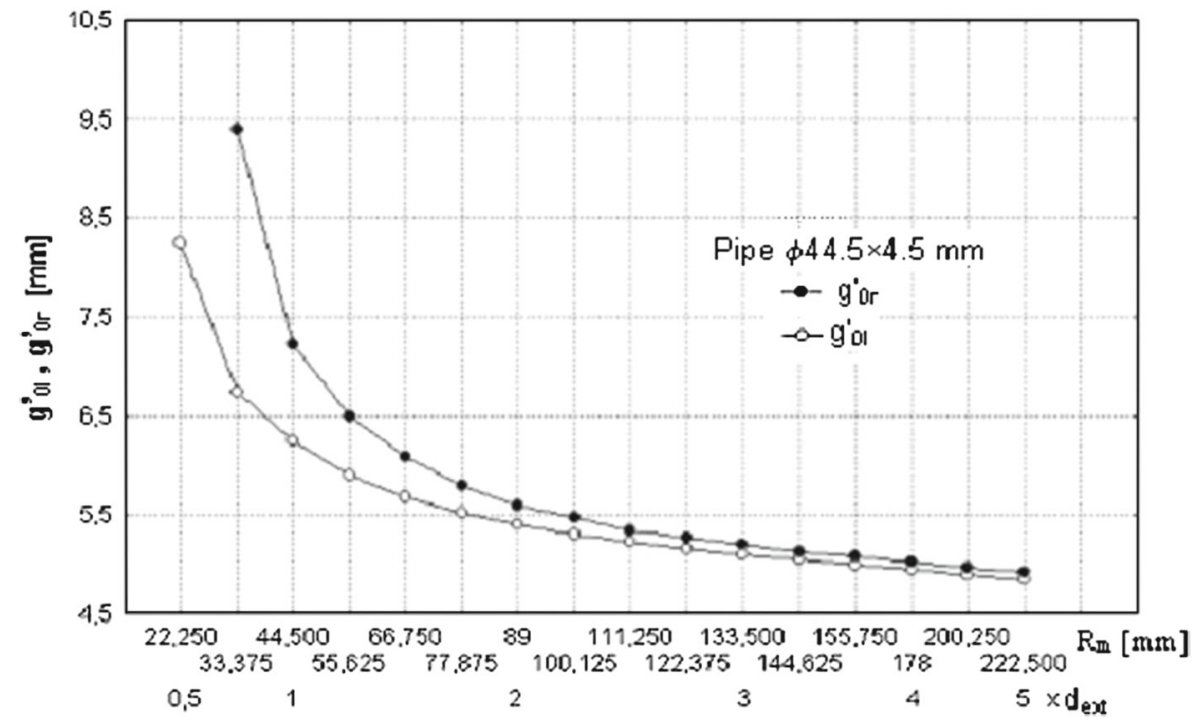

Fig. 4 Variation of the initial wall thickness $\left(g_{0 r}^{\prime}, g_{0 l}^{\prime}\right)$ with bending radius $R_{\mathrm{m}}$, if a required minimum wall thickness of a bend for the pipe $d_{\text {ext }}=44.5 \mathrm{~mm}$ is $g_{1}=4.5 \mathrm{~mm}$

one should use the measures of relative strain and not the measures of logarithmic strain. It is recommended to do so, despite the fact that the measures of logarithmic strain are very useful and frequently used and more specifically describe the states of strain in several forming processes and during tube bending [10-13,17-24].

Graphs of initial thicknesses of the bent pipes depending on the value of the average bending radius $R_{\mathrm{m}}$, when the required (desired) thickness of the wall of the bent knee is $g_{1}=4.5 \mathrm{~mm}$, are presented in Table 2 and Fig. 4.

Analogously to the graph presented in Table 1 and Fig. 3, when the bending radius $R_{\mathrm{m}}$ decreases, the differences in calculated wall thicknesses increase and vice versa. When bending radius $R_{\mathrm{m}}$ increases, then strains decrease and both measures of strain became nearly equal. When using the reverse dependencies, derived at this point (which are used to determine the initial thickness of the pipe to be bent), the application of the measures of the relative strain determines larger, and therefore more secure values applied to $g_{0}$ than the 
application of real measures of strain. At the end, it should be mentioned that derived in this work inequalities (12)-(15) and (18) will be also met for each bending radius $R_{\mathrm{m}}$ and all geometric dimensions of bent tube and for $\left(R>y_{0 \max }\right)$.

It was shown in papers $[10,12,13]$ that the use of logarithmic (real) measures of strain very well describes (even with accuracy of about $1 \%$ ) experimental data found in [17-21] and own authors' data, in both layers, stretched and compressed. On graphs in Figs. 3 and 4, the results of respective calculations for $\left(g_{1 r}^{\prime}\right.$ and $\left.g_{0 r}^{\prime}\right)$ were compared, obtained using relative measures of strain in stretched layers (according to EU-Directive [1,2]) with those for $\left(g_{1 l}^{\prime}\right.$ and $\left.g_{0 l}^{\prime}\right)$ obtained using logarithmic measures. It means that, in fact, with accuracy of a few percent, computational data resulting from expression included in EU-Directive with experimental data included in $[17,20]$ were compared. This comparison leads to conclusion that strains calculated using the EU-Directive are greater than the real ones and wall thicknesses in stretched layers are smaller. Therefore, the respective initial wall thicknesses of the tube to be bent are larger than the thicknesses resulting from assuming the measures of logarithmic strain. These estimations will be safer taking into account ultimate and temporary strength, service life, and safety of working conditions.

Adoption of first-order simplifications of logarithmic and relative measures of strain provides, in addition to the advantages mentioned previously, very good accuracy of description of experimental data. These simplifications also take into account, during real processes of bending tubes on benders, the effect of even lowering with the angle of bending and mutually proportional (due to the effects of thinning and ovality of cross section) of the outer stretched layers and simultaneous shifting "downwards" (in the direction of the center of curvature and rotation) (see e.g., [10-13,17-24]) inert layer of plastic bending. During the bending of tubes on a mandrel with a trackpad and using a profiled strip with an adjustable clamp and with minimum clearances between the tools and the walls of the bent pipe, plastically deformed material of pipe will move more "sideways" and less swell in compression layers, which will cause that actual position of the inert layer will be less moved "downwards" (see e.g., [10-13,17-24]). Due to the occurrence of these effects, in real technological processes of bending pipes, we have some physical justification for the use of simplified expressions of first degree. The simplifications in the formulas for the longitudinal (axial) strain thus contain in numerator the value of $d_{\text {ext }}$ instead of $d_{i}$.

\section{Notes on choosing of tube bending technology}

According to EU-Directive [1,2], the required minimum wall thickness of the bent knee in the stretched layers (for pipes of a nominal outer diameter $d_{\mathrm{ext}} \leq 142 \mathrm{~mm}$ ) is basically determined by the following formula

$$
g_{1 \text { req }}=g_{0} \frac{4 R_{\mathrm{m}}+d_{\mathrm{ext}}}{4 R_{\mathrm{m}}+2 d_{\mathrm{ext}}},
$$

where $g_{1 \text { req }}$-the required minimum wall thickness of the bent knee in stretched layers.

For tubes with outer diameter $\left(80 \mathrm{~mm} \leq d_{\mathrm{ext}} \leq 142 \mathrm{~mm}\right.$ ), the required minimum wall thickness from the inner side (in compressed layers) should not be smaller than determined by formula:

$$
g_{2 \text { req }} \geq g_{0} \frac{4 R_{\mathrm{m}}-d_{\mathrm{ext}}}{4 R_{\mathrm{m}}-2 d_{\mathrm{ext}}},
$$

where $g_{2 \text { req }}$-the required minimum wall thickness in compressed layers.

As it results from formulas (19), (20), published in EU-Directive, they take into account the shift of the neutral axis of plastic bending, since $R \neq R_{\mathrm{m}}$, then $y_{0} \neq 0$.

In order to obtain the knees corresponding to the requirements of EU-Directive, one can also change the technology (method) of tube bending for another, for example for bending tubes on benders with pushing and using the appropriate template and mandrel (see e.g., [17,19-21]. Separate studies are required in order to choose the stiffness of bending machine and adequate clearances between a bent tube and tools (instrumentation) and the way of mutual synchronization of frequency of pushing force with the rotation of template.

\section{Remarks and conclusions}

1. The above examples of computational results show that the relative strain and the required minimum wall thickness calculated according to the EU-Directive are higher (for small strains) than those obtained with 
the use of logarithmic strain measures (real). On the other hand, logarithmic measures of strain, due to their practical properties, are most often applied to the description of strain state in many forming processes, including tube bending (see e.g., [10-13,17-21]). This fact may cause some problems in designing and technology and also in strength and operation.

2. The corresponding expressions for the intensity of plastic deformation and incompressibility of plastic material for large deformations in relative measures of deformations have the form

$$
\left\{\begin{array}{l}
\varepsilon_{(i)}^{\prime}=\exp \sqrt{\frac{2}{3}\left(\ln ^{2}\left(1+\varepsilon_{1}^{\prime}\right)+\ln ^{2}\left(1+\varepsilon_{2}^{\prime}\right)+\ln ^{2}\left(1+\varepsilon_{3}^{\prime}\right)\right)}-1, \\
\varepsilon_{1}^{\prime}+\varepsilon_{2}^{\prime}+\varepsilon_{3}^{\prime}+\varepsilon_{1}^{\prime} \varepsilon_{2}^{\prime}+\varepsilon_{1}^{\prime} \varepsilon_{3}^{\prime}+\varepsilon_{2}^{\prime} \varepsilon_{3}^{\prime}+\varepsilon_{1}^{\prime} \varepsilon_{2}^{\prime} \varepsilon_{3}^{\prime}=0 .
\end{array}\right.
$$

If we take expression $(21)_{2}$ instead of $(9)_{1}$, then expressions for $g_{1 r}^{\prime}$ and $g_{0 r}^{\prime}$ will have different, more composed formulae, but inequalities (14) 1 and (15) will be still valid.

3. In order to obtain the required (in accordance with EU-Directive concerning pressure equipment and its production, included in [1]) values of strain and the thickness of bent knees and the initial wall thickness of tubes to be bent for small deformations, we should use the relations (6), (11) $)_{1}$, and (16) derived in this work and $(9)_{1}$. However, for precise solution within continuum mechanics for large deformations, we should use the relations (6) and (21).

4. This work can be treated as the first step, and the next steps could be development of nomograms and tables for bending tubes of various dimensions $\left(d_{\mathrm{ext}} \times g_{0}\right)$ or $\left(d_{\mathrm{int}} \times g_{0}\right)$ for various $R_{\mathrm{m}}$ applying expressions (6) and (21), valid for large deformations. When initial thickness $g_{0 l}$ or $g_{0 r}$ are calculated depending on $d_{\mathrm{ext}}$ or $d_{\text {int }}$, the results are different, see [11]. EU-Directive contains dependence on $d_{\text {ext }}$ not on $d_{\text {int }}$.

5. The solution of the problem of pipe bending on benders in the framework of nonlinear solid mechanics is difficult due to complex relations between stresses and deformations, see [26-31] and is open to further studies. We should remember that tube bending is not a free process but depends on bender, its stiffness, shape of working tools, type of applied mandrels, bending parameters such as $R_{\mathrm{m}}$, tube dimensions $\left(d_{\mathrm{ext}} \times\right.$ $\left.g_{0}\right)$, tube material, and others [1,2,9-14,17-24].

Open Access This article is distributed under the terms of the Creative Commons Attribution License which permits any use, distribution, and reproduction in any medium, provided the original author(s) and the source are credited.

\section{References}

1. EN 13445-4 Unfired Pressure Vessels_Part 4: Fabrication, (point 9.2.4 Tube Bents, expression 9.2-4), (2009) or earlier Draft Standart EN UFPV "Unfired Pressure Vessels"-Part 4. "Manufacture" (May 1996) CEN/TC54/267 JWGB N277 rev. 5

2. Zdankiewicz, M.: European Directive Concerning Pressure Installations. Requirements Concerning Manufacturing [in Polish]. Technical Inspection (Dozór Techniczny), vol. 2, pp. 25-33 and 48 (1998)

3. Gabryszewski, Z.: Theory of Elasticity and Plasticity [in Polish], Publishing House of Wrocław University of Technology Wrocław (2001)

4. Hill, R.: Mathematical Theory of Plasticity. Clarendon Press, Oxford (1985)

5. Johnson, W., Mellor, P.B.: Engineering Plasticity. van Nostrand Reinhold Company, London (1975)

6. Lubliner, J.: Plasticity Theory. Macmillan, New York (1990)

7. Mendelson, A.: Plasticity-Theory and Applications. Mc Millan Company, New York (1988)

8. Olszak, W., Perzyna, P., Sawczuk, A.: Theory of Plasticity. PWN, Warszawa (1981)

9. Życzkowski, M.: Combined Loading in the Theory of Plasticity. PWN-Nijhoff, Warszawa-Alpen aan den Rijn (1981)

10. Śloderbach, Z., Strauchold, Sz.: Approximate Methods for Evaluating Strains in Pipe Bending Processes [in Polish]. Technical Inspection (Dozór Techniczny), vol. 1, pp. 1-6 (1999)

11. Śloderbach, Z:: Some Problems of Mechanics in Pipeline Bending Processes [in Polish], Publishing House of Wrocław University of Technology, Wrocław (2002)

12. Śloderbach, Z.: Generalized model of strains during bending of metal tubes in bending machines. J. Theor. Appl. Mech. 52(4), 1093-1106 (2014)

13. Śloderbach, Z.: A model of deformation geometry in pipe bending processes. Eng. Trans. IFTR-PAS 47(1), 3-20 (1999)

14. Śloderbach, Z., Rechul, Z.: Effect of strain hardening and normal anisotropy on allowable values of strain and stress in pipe-bending processes. J. Theor. Appl. Mech. 38(4), 843-859 (2000)

15. Team Work Technical Requirement. Calculations and checking of wall thickness of bent tubes [in Polish], Steam Boilers Factory "RAFAKO” S.A., WT-1/0/R, Racibórz (1991)

16. Team Work Pressure Installations. General Requirements. Strength Calculations [in Polish], UDT Conditions, (WUDT-UCWO-O/02:10) Issue I, Warszawa (2003) 
17. Franz, W.D.: Das Kalt-Biegen von Rohren. Springer, Berlin (1961)

18. Franz, W.D.: Numerisch gesteuerte Rohrkaltbiegemaschinen. Werkstatt Und Betrieb 9, 129-145 (1969)

19. Grunow, O.: Praktisches Rohrbiegen. Springer, Berlin (1985)

20. Korzemski, J.W.: Thin-Walled Pipe Bending [in Polish]. WNT, Warszawa (1971)

21. Wick, Ch., Benedict, J.T., Veilleux, R.F.: Tool and Manufacturing Engineers Handbook. A Reference Book for Manufacturing Engineers, Managers and Technicians, Forming, 4th Edn., vol. II. Society of Manufacturing Engineers, One SME Drive, Dearbon, Michigan, USA (2001)

22. Zhang, Z., Yang, H., Li, H., Ren, N., Tian, Y.: Bending behaviors of large diameter thin walled CP-Ti tube in rotary draw bending. Prog. Nat. Sci.: Mater. Int. 21, 401-412 (2011)

23. Zhiqiang, J., Mei, Z., He, Y., Xudong, X., Guangjun, L.: Deformation behavior of medium strength TA18 high-pressure tubes during NC bending, with different bending radii. Chin. J. Aeronaut. 24, 657-664 (2011)

24. Tang, N.C.: Plastic-deformation analysis in tube bending. Int. J. Press. Vessel. Pip. 77(12), 751-759 (2000)

25. Szczepiński, W.: Theory of Plastic Working of Metals [in Polish]. PWN, Warszawa (1987)

26. Gabryszewski, Z., Gronostajski, J.: Fundamentals of Metal-Working Processes [in Polish]. PWN, Warszawa (1991)

27. Pęcherski, R.B.: Finite deformation plasticity with strain induced anisotropy and shear banding. J. Mater. Process. Technol. 60, 35-44 (1996)

28. Haupt, P.: Continuum Mechanics and Theory of Materials. Springer, Berlin (2002)

29. Huttel, C., Matzenmiller, A.: Extension of generalized plasticity to finite deformations and structures. Int. J. Plast. 36, 5255$5276(1999)$

30. Śloderbach, Z., Pajak, J.: Generalized coupled thermoplasticity taking into account large strains: part I. Conditions of uniqueness of the solution of boundary-value problem and bifurcation criteria. Math. Mech. Solids 15(3), 308-327 (2010)

31. Życzkowski, M., Szuwalski, K.: On the termination of the process of finite plastic deformations. J. Mécanique Theoretique et Applique 1, Numero special 175-186 (1982) 